Arq. Bras. Med. Vet. Zootec., v.56, n.1, p.81-85, 2004

\title{
Erros de anotações na elaboração de índices de produção em granjas industriais de suínos no Sul do Brasil
}

\author{
[The human effect on data collection of birth-related production indices in \\ Brazilian swine industrial farms] \\ L.G. Schneider ${ }^{1}$, F.P. Bortolozzo ${ }^{2}$, I. Wentz $^{2}$, G. Borchardt Neto ${ }^{3}$ \\ ${ }^{1}$ Médico Veterinário, MSc \\ ${ }^{2}$ Setor de suínos - UFRGS - Porto Alegre, RS \\ ${ }^{3}$ Laboratório de Reprodução Animal - Universidade de Cruz Alta - Cruz Alta, RS
}

\begin{abstract}
RESUMO
Foram realizadas observações em quatro granjas de suinocultura industrial nas 24 horas do dia, registrando-se o número de fetos mumificados, de natimortos, de nascidos vivos e total segundo as observações feitas por funcionários da granja (FUN) e por pesquisadores (AO). Foram também registrados resultados de um número semelhante de anotações das mesmas características no período imediatamente anterior à pesquisa, obtidas dos relatórios das granjas. Verificaram-se diferenças entre as avaliações dos FUN e dos AO quanto aos registros dos mumificados e total de nascidos em todas as granjas $(\mathrm{P}<0,05)$. A diferença no número de natimortos ocorreu em três granjas $(\mathrm{P}<0,05)$ e de nascidos vivos em apenas uma $(\mathrm{P}<0,05)$. Os percentuais máximos de mumificados, de natimortos, de nascidos vivos e total não registrados pelos FUN foram $67,8 \%, 34,5 \%, 1,8 \%$ e 5,1\%, respectivamente. Os percentuais máximos das mesmas características não registrados pelos FUN no período imediatamente anterior à pesquisa foram $70,4 \%, 82,1 \%, 10,5 \%$ e $16,5 \%$.
\end{abstract}

Palavras-chave: erros de registro, mumificados, natimortos, leitegada

\begin{abstract}
Observational studies were carried out in four industrial swine farms. The observations were performed on a 24 hours basis and mummified fetuses, stillborn and born alive piglets were recorded by observers $(O B)$ and by employees (EM). In all farms, it was also obtained a retrospective data previous to the observational study period, comprising the same number of farrowings. Differences among mummified fetuses and total born piglets recorded by $O B$ and EM in all farms $(P<0.05)$ were observed. The recorded number of stillborn piglets differed between $O B$ and EM in three farms $(P<0.05)$ and the number of born alive piglets in one unit $(P<0.05)$. In all farms during the analysis period, the maximal percentage of recorded mummified fetuses, stillborn, born alive and total born piglets, were $67.8 \%, 34.5 \%, 1.8 \%$ and $5.1 \%$, respectively. The comparison of retrospective and EM data showed a maximal percentage of not recorded mummified fetuses, stillborn, born alive and total born piglets by EM of $70.4 \%, 82.1 \%, 10.5 \% \mathrm{e}$ $16.5 \%$, respectively. These errors can induce to a wrong interpretation of the farm data and the efforts to maximize the productivity could be directed to other sectors than the farrowing house.
\end{abstract}

Keywords: record collection errors, mummified, fetuses, stillbirth, litter

Recebido para publicação em 26 de julho de 2002

Recebido para publicação, após modificações, em 1 de setembro de 2003

*Endereço para correspondência

Rua Tarquíneo Oliveira, 77, Bairro Cruzeiro

98900-000 - Santa Rosa, RS,

E-mail: schneider@vitagri.com.br 


\section{INTRODUÇÃO}

A suinocultura industrial moderna busca, cada vez mais, o aumento da produtividade, a qual pode ser mensurada por vários parâmetros, dentre os quais, o número de leitões desmamados/fêmea/ano é um dos mais utilizados (Dial et al., 1992; Muirhead, Alexander, 1997; English, 1998; Okere, 1999).

Para que seja atingida a produtividade satisfatória em uma unidade de suínos torna-se essencial que todas as atividades desenvolvidas na granja estejam bem sincronizadas, atendendo às técnicas de manejo recomendadas, com adequado treinamento de funcionários, e que o sistema de coleta de dados, fundamental para a formação exata dos índices de produção, seja correto (Dial et al., 1992; Muirhead, Alexander, 1997; Lucia et al., 1999).

Na prática, a interpretação correta dos índices de produção constitui o primeiro aspecto a ser considerado em uma visita técnica à granja, para que sejam desenvolvidas, se necessárias, estratégias adequadas de manejo (Muirhead, Alexander, 1997). Estudos demonstram que os registros de dados, os quais formam os índices de produção, podem ser elaborados de forma errada. Essa condição foi relativamente bem caracterizada por Vaillancourt et al. (1990). Esses autores estudaram as causas de mortalidade pré-desmame em suínos e identificaram diferenças na acurácia dos registros realizados por produtores e funcionários (FUN). Os valores variaram de 35,5 a 95,7\% em 13 granjas comerciais examinadas. Apesar dos erros em termos de diagnóstico terem sido bem estudados, as falhas de registros relacionadas à quantidade de leitões ao parto, por exemplo, foram uma constante. $\mathrm{O}$ objetivo deste trabalho foi avaliar a influência do ser humano na elaboração dos índices de produção relacionados ao parto em granjas de suínos.

\section{MATERIAL E MÉTODO}

Foram realizadas observações em quatro granjas com suinocultura tecnificada por uma equipe de três pesquisadores. As granjas 1 e 2, unidades produtoras de leitões, com 2.400 e 3.600 fêmeas, respectivamente, estão localizadas no Noroeste gaúcho; a granja 3, unidade de ciclo completo, com 1.200 matrizes, situa-se na região Sudoeste gaúcha; a granja 4, unidade produtora de leitões, com 2.200 matrizes, está localizada no Oeste paranaense. As observações foram realizadas nas 24 horas do dia, durante duas semanas, em cada unidade de produção. Os partos foram acompanhados sem que houvesse interferência na rotina de trabalho da maternidade, anotandose todas as ocorrências.

Ao final do parto foram registrados o número de: fetos mumificados (MUM), leitões natimortos (NAT), vivos e total (MUM + NAT + vivos). As anotações dos funcionários atendentes dos partos e da equipe de pesquisa foram com paradas. Previamente à realização das observações, supôsse que a presença de pessoas estranhas ao ambiente de trabalho na maternidade (pesquisadores) poderia inibir os funcionários quanto ao cumprimento de suas obrigações, como por exemplo, registrar a taxa de leitões natimortos ao parto. Essa variável de interferência foi designada efeito de pessoas estranhas ao ambiente de trabalho (PEAT). Sua conseqüência poderia ser a diminuição da diferença entre os registros dos funcionários da granja e dos pesquisadores.

Para a boa interpretação dos resultados e talvez justificar o efeito PEAT, os resultados obtidos pela equipe de pesquisa no período do experimento foram também comparados com os registros contidos nos relatórios dos FUN, no período imediatamente anterior ao do experimento, porém a comparação foi apenas descritiva.

As diferenças entre os registros quanto aos MUM, NAT, leitões nascidos vivos e total foram comparadas pelo teste $\mathrm{t}$ para amostras pareadas (User's..., 1996).

\section{RESULTADOS E DISCUSSÃO}

Os registros de MUM realizados pelos funcionários (FUN) e pelos pesquisadores (AO) diferiram em todas as granjas $(\mathrm{P}<0,05)$ (Tab. 1). Por exemplo, a média de fetos MUM/leitegada segundo os FUN variou de 0,09 (granja 1) a 0,52 (granja 4), e segundo os pesquisadores, de 0,28 (granja 1) a 0,71 (granja 4). Isto indica que não foram registrados $67,8 \%$ dos fetos MUM na granja 1 e $26,8 \%$ na granja $4(\mathrm{P}<0,05)$. Com 
relação aos leitões NAT, as falhas de anotação ocorreram nas quatro granjas, porém em menor porcentagem. A média de leitões NAT registrados pelos FUN variou de 0,72 (granja 1) a 1,05 (granjas 4), e pelos pesquisadores, de 1,10 (granja 1) a 1,12 (granja 4). Isto indica o não registro de $34,5 \%$ e $6,2 \%$ de natimortos nas granjas 1 e 4 , respectivamente.

Tabela 1. Número de fetos mumificados e de leitões natimortos segundo os registros dos funcionários da granja (FUN) e dos pesquisadores (AO), durante o período de estudo em granjas de suínos

\begin{tabular}{|c|c|c|c|c|c|c|c|c|c|}
\hline \multirow{3}{*}{ Granja } & \multirow{3}{*}{$\mathrm{N}$} & \multicolumn{4}{|c|}{ Mumificados/leitegada } & \multicolumn{4}{|c|}{ Natimortos/leitegada } \\
\hline & & \multirow[b]{2}{*}{ FUN } & \multirow[b]{2}{*}{$\mathrm{AO}$} & \multicolumn{2}{|c|}{ Diferença } & \multirow[b]{2}{*}{ FUN } & \multirow[b]{2}{*}{$\mathrm{AO}$} & \multicolumn{2}{|c|}{ Diferença } \\
\hline & & & & $\mathrm{n}$ & $\%$ & & & $\mathrm{n}$ & $\%$ \\
\hline 1 & 142 & $0,09 \pm 0,3$ & $0,28 \pm 0,6$ & $-0,19^{*}$ & 67,8 & $0,72 \pm 1,0$ & $1,10 \pm 1,2$ & $-0,38^{*}$ & 34,5 \\
\hline 2 & 168 & $0,11 \pm 0,4$ & $0,27 \pm 0,7$ & $-0,16^{*}$ & 59,2 & $0,77 \pm 1,2$ & $0,83 \pm 1,1$ & $-0,06$ & 7,2 \\
\hline 3 & 116 & $0,21 \pm 0,6$ & $0,26 \pm 0,6$ & $-0,05^{*}$ & 19,2 & $0,95 \pm 1,4$ & $1,12 \pm 1,5$ & $-0,17^{*}$ & 15,2 \\
\hline 4 & 131 & $0,52 \pm 1,3$ & $0,71 \pm 1,3$ & $-0,19^{*}$ & 26,8 & $1,05 \pm 1,2$ & $1,12 \pm 1,3$ & $-0,07^{*}$ & 6,2 \\
\hline
\end{tabular}

Quanto aos registros do número de leitões nascidos vivos (Tab. 2), somente na granja 2 houve diferença entre as contagens dos FUN e dos AO $(1,8 \% ; \mathrm{P}<0,05)$. Na granja 4 , o número (não significativo) de nascidos registrados pelos FUN foi maior do que os registrados pelos AO. Os erros de registros do total de leitões nascidos persistiram em todas as granjas $(\mathrm{P}<0,05)$, semelhante ao ocorrido com os MUM. Não foram registrados $5,1 \%$ dos leitões na granja 1 , refletindo as falhas cometidas em outras categorias de leitões, porém em menor porcentagem.

Tabela 2. Leitões nascidos vivos e total segundo os registros dos funcionários da granja (FUN) e dos pesquisadores $(\mathrm{AO})$, durante o período de estudo em granjas de suínos

\begin{tabular}{|c|c|c|c|c|c|c|c|c|c|}
\hline \multirow{3}{*}{ Granja } & \multirow{3}{*}{$\mathrm{N}$} & \multicolumn{4}{|c|}{ Nascidos vivos/leitegada $( \pm \mathrm{DP})$} & \multicolumn{4}{|c|}{ Total nascidos/leitegada $( \pm \mathrm{DP})$} \\
\hline & & \multirow[b]{2}{*}{ FUN } & \multirow[b]{2}{*}{$\mathrm{AO}$} & \multicolumn{2}{|c|}{ Diferença } & \multirow[b]{2}{*}{ FUN } & \multirow[b]{2}{*}{$\mathrm{AO}$} & \multicolumn{2}{|c|}{ Diferença } \\
\hline & & & & $\mathrm{n}$ & $\%$ & & & $\mathrm{n}$ & $\%$ \\
\hline 1 & 142 & $10,62 \pm 2,7$ & $10,78 \pm 2,5$ & $-0,16$ & 1,5 & $11,54 \pm 2,8$ & $12,16 \pm 2,9$ & $-0,62^{*}$ & 5,1 \\
\hline 2 & 168 & $9,87 \pm 2,9$ & $10,05 \pm 2,8$ & $-0,18^{*}$ & 1,8 & $10,79 \pm 3,0$ & $11,16 \pm 3,2$ & $-0,37^{*}$ & 3,3 \\
\hline 3 & 116 & $10,00 \pm 3,1$ & $10,10 \pm 3,0$ & $-0,10$ & 0,1 & $11,25 \pm 3,4$ & $11,53 \pm 3,5$ & $-0,28^{*}$ & 2,4 \\
\hline 4 & 131 & $10,47 \pm 3,1$ & $10,40 \pm 3,2$ & 0,07 & $-0,7$ & $12,00 \pm 3,8$ & $12,33 \pm 3,9$ & $-0,33^{*}$ & 2,7 \\
\hline
\end{tabular}

As Tab. 3 e 4 apresentam os mesmos dados relacionados pelas Tab. 1 e 2 , porém os registros dos FUN foram os anteriores ao período de avaliação feito pelos AO. Estes resultados não foram submetidos à analise estatísticas e são apresentados apenas como uma contribuição para estudos futuros, com o objetivo de evitar o efeito PEAT. Observa-se que os erros nos registros podem ser, supostamente, mais pronunciados para todas as características estudadas.

Tabela 3. Número de fetos mumificados e de leitões natimortos segundo o registro dos funcionários da granja (FUN) em período anterior ao estudo, e segundo os pesquisadores (AO), em granjas de suínos

\begin{tabular}{|c|c|c|c|c|c|c|c|c|c|}
\hline \multirow{3}{*}{ Granja } & \multirow{3}{*}{$\mathrm{N}$} & \multicolumn{4}{|c|}{ Mumificados/leitegada } & \multicolumn{4}{|c|}{ Natimortos/leitegada } \\
\hline & & \multirow[b]{2}{*}{ FUN } & \multirow[b]{2}{*}{$\mathrm{AO}$} & \multicolumn{2}{|c|}{ Diferença } & \multirow[b]{2}{*}{ FUN } & \multirow[b]{2}{*}{$\mathrm{AO}$} & \multicolumn{2}{|c|}{ Diferença } \\
\hline & & & & $\mathrm{n}$ & $\%$ & & & $\mathrm{n}$ & $\%$ \\
\hline 1 & 142 & 0,13 & 0,28 & $-0,15$ & 53,6 & 0,37 & 1,10 & $-0,73$ & 66,3 \\
\hline 2 & 168 & 0,08 & 0,27 & $-0,19$ & 70,4 & 0,33 & 0,83 & $-0,50$ & 60,2 \\
\hline 3 & 116 & 0,10 & 0,26 & $-0,16$ & 61,5 & 0,20 & 1,12 & $-0,92$ & 82,1 \\
\hline 4 & 131 & 0,31 & 0,71 & $-0,40$ & 56,3 & 0,59 & 1,12 & $-0,53$ & 47,3 \\
\hline
\end{tabular}


Tabela 4. Leitões nascidos vivos e total segundo os registros dos funcionários da granja (FUN) em período anterior ao estudo, e segundo os pesquisadores (AO), em granjas de suínos

\begin{tabular}{|c|c|c|c|c|c|c|c|c|c|}
\hline \multirow{2}{*}{ Granja } & \multirow{2}{*}{$\mathrm{N}$} & \multicolumn{4}{|c|}{ Nascidos vivos/leitegada } & \multicolumn{4}{|c|}{ Nascidos totais/leitegada } \\
\hline & & FUN & $\mathrm{AO}$ & DN & $\mathrm{D} \%$ & FUN & $\mathrm{AO}$ & $\mathrm{DN}$ & $\mathrm{D} \%$ \\
\hline 1 & 142 & 9,65 & 10,78 & $-1,13$ & 10,5 & 10,15 & 12,16 & $-2,01$ & 16,5 \\
\hline 2 & 168 & 9,50 & 10,05 & $-0,55$ & 5,5 & 10,00 & 11,16 & $-1,16$ & 10,4 \\
\hline 3 & 116 & 9,60 & 10,10 & $-0,50$ & 4,9 & 9,90 & 11,53 & $-1,63$ & 14,1 \\
\hline 4 & 131 & 10,40 & 10,40 & 0 & 0 & 11,30 & 12,33 & $-1,03$ & 8,3 \\
\hline
\end{tabular}

Na literatura consultada não foram encontrados estudos relatando erros qualitativos e quantitativos nos registros dos leitões nascidos. Alguns autores sugeriram que essas falhas podem fazer com que a interpretação dos resultados de desempenho dos rebanhos seja equivocada (Blackwell, 1987; Lucia et al., 1999).

A variável MUM foi a que mais apresentou falhas nos registros. Os erros são, possivelmente, causados por falhas na identificação dos fetos ou pela ausência de registro dos MUM de tamanho reduzido, que podem estar envolvidos na placenta, principalmente pelo desconhecimento da importância do seu registro. Segundo Muirhead e Alexander (1997), fetos suínos com 30 a 50 dias de gestação têm de 20 a $88 \mathrm{~mm}$ de comprimento, o que dificulta sua identificação. As falhas na anotação dos NAT podem ser intencionais, na tentativa de reduzir o seu índice, com o objetivo de atingir uma determinada meta pré-estabelecida para essa característica. Segundo Blackwell (1987), o não registro de leitões natimortos pode dificultar o controle da natimortalidade, ao determinar a taxa de natimortos abaixo da realidade.

As diferenças entre os registros dos FUN e dos AO podem estar subestimadas, pelo fato de não se analisar o efeito PEAT. Os resultados efetivamente registrados pelos FUN sem a presença de estranhos, possivelmente refletem melhor os acontecimentos diários da granja. Leitões nascidos vivos, porém pequenos e com baixa viabilidade, podem não ser registrados, e, caso sobrevivam, são incorporados ao registro de outra fêmea ou de leitões que foram registrados e morreram mais tarde, na lactação. Outra causa do não apontamento de leitões nascidos vivos refere-se à tentativa de manipulação para controle da taxa de mortalidade pré-desmame. Somente na granja 2 foi observada diferença no número médio de leitões nascidos vivos registrados pelos FUN e pelos AO. Na granja 4, os números foram até favoráveis, o que demonstra a excelente qualidade das anotações para leitões nascidos vivos feitas pelos FUN. Quanto ao número total de leitões nascidos, também se observaram erros de registro. Em todas as características há evidências de que o efeito PEAT deve ser minimizado. Vale salientar que os índices registrados no período anterior ao da pesquisa foi semelhante ao observado nas unidades, ao longo dos 12 meses que precederam o estudo. A comparação entre o período prévio e o período de observação efetivo foi a melhor maneira encontrada para minimizar o efeito PEAT.

Os erros de registro no número de leitões nascidos tendem a subestimar o tamanho da leitegada (total de nascidos). Ao realizar uma intervenção em uma unidade de produção de suínos com leitegadas pequenas dirija seus esforços para outros setores de produção além da maternidade, pois em algumas a ocorrência de MUM, NAT e as perdas pré-desmame podem estar controladas.

\section{CONCLUSÕES}

Os erros de registro do número de leitões nascidos ao parto cometidos pelos funcionários das granjas podem ser acentuados, dependendo da unidade de produção avaliada. Ao minimizar o efeito de pessoas estranhas ao ambiente de trabalho sobre a qualidade dos registros, os erros podem ser, supostamente, mais pronunciados. Os erros discutidos dificultam a assistência técnica nas granjas, pela interpretação de índices de produção irreais.

\section{REFERÊNCIAS BIBLIOGRÁFICAS}

BLACKWELL, T.E. Predicting stillbirth problems. Swine production management. Comp. Food Anim., v.9, p.F371-F374, 1987. 
DIAL, G.D.; MARSH, W.E.; POLSON, D.D. et al. Reproductive failure: differential diagnosis. In: LEMAN, A.D.; STRAW, B.E.; MENGELING, W.L. et al. Diseases of swine. London: Wolfe, 1992. p.88-137.

ENGLISH, P.R. Improving reproductive performance of the breeding herd and the education, training and motivation of stockpeople. In: SEMINÁRIO INTERNACIONAL DE SUINOCULTURA, 3., 1998, São Paulo. Anais... São Paulo: [s.n.], 1998. p.85-100.

LUCIA, T.J.R.; DESCHAMPS, J.C.; CORRÊA, M.N. Estratégias de gerenciamento de informação aplicadas à suinocultura. Rev. Bras. Reprod. Anim., v.23, p.132-140, 1999.
MUIRHEAD, M.R.; ALEXANDER, T.J.L. Managing pig health and the treatment of disease. United Kingdom: 5M, 1997. 608p.

OKERE, C. Stillbirths: unraveling a complex problem. Tech. Ser. Gen. Swine Gr., v.4, p.1-6, 1999.

USER'S guide: statistical analysis system. Release 6.11. Cary, NC: SAS Institute,1996.

VAILLANCOURT, J.P.; STEIN, T.E.; MARSH, W.E. et al. Validation of producer-recorded causes of preweaning mortality in swine. In: CONGRESS OF INTERNATIONAL PIG VETERINARY SOCIETY, 11., Lauzanne, 1990. Proceedings..., Lauzanne: [s.n.], 1990. p.386. 